\title{
High Risk of Tumor Lysis Syndrome in Symptomatic Patients with Multiple Myeloma with Renal Dysfunction Treated with Bortezomib
}

\author{
KANA OIWA $^{1 *}$, MIHOKO MORITA ${ }^{1 *}$, SHINJI KISHI ${ }^{1,2}$, MIYUKI OKURA $^{1}$, \\ TOSHIKI TASAKI ${ }^{1}$, YASUFUMI MATSUDA ${ }^{1}$, KATSUNORI TAI $^{1}$, \\ NAOKO HOSONO ${ }^{1}$, TAKANORI UEDA ${ }^{1}$ and TAKAHIRO YAMAUCHI ${ }^{1}$ \\ ${ }^{1}$ Department of Hematology and Oncology, University of Fukui, Fukui, Japan; \\ ${ }^{2}$ Department of Health and Nutrition, Faculty of Human Life Studies, Jin-ai University, Fukui, Japan
}

\begin{abstract}
Background/Aim: Tumor lysis syndrome (TLS) is a life-threatening complication associated with cancer chemotherapy. We retrospectively evaluated the risk of developing TLS in patients with symptomatic multiple myeloma undergoing chemotherapy. Patients and Methods: Sixty-four patients (median age $=71$ years, range $=48-87$ years, 35 males/29 females) who were treated at our Institution between April 2006 and December 2015 were evaluated. Results: A total of 124 chemotherapy courses were administered, of which 63 courses were bortezomib-based regimens and 34 courses were immunomodulatory drug (IMiD)-based regimens. TLS occurred in 13 (10.5\%) out of 124 chemotherapy courses with five $(4.0 \%)$ cases of laboratory TLS and eight (6.5\%) cases of clinical TLS. The incidences of TLS were $17.5 \%$ for bortezomib-containing regimens and 3.2\% for non-bortezomibbased regimens. No TLS occurred in the patients treated with IMiD-containing regimens. TLS occurred more frequently in the patients with elevated uric acid, creatinine, or beta-2microglobulin levels at baseline. The patients with disease classified as advanced International Staging System also developed TLS more frequently. All the patients who developed clinical TLS received bortezomib-containing regimens $(8 / 63$, $12.7 \%$ ). Among them, patients with elevated values of uric acid or creatinine developed clinical TLS more often than those
\end{abstract}

This article is freely accessible online.

*These Authors contributed equally to the present study.

Correspondence to: Shinji Kishi, Department of Hematology and Oncology, University of Fukui, 23-3, Shimoaizuki, Matsuoka, Eiheiji, Fukui 910-1193, Japan. Tel: +81 776613111, Fax: +81 776618109, e-mail: skishi@u-fukui.ac.jp

Key Words: Tumor lysis syndrome, symptomatic myeloma, bortezomib, renal dysfunction. without such elevation. The incidence of clinical TLS was $33.3 \%$ if the patients had renal dysfunction at baseline and were subsequently treated with bortezomib-based regimens (8/24 cases). Conclusion: Patients with renal dysfunction or a high uric acid level receiving bortezomib-based chemotherapy have a high risk of developing TLS.

Tumor lysis syndrome (TLS) is a life-threatening complication associated with cancer chemotherapy for highly proliferative malignancies (1). TLS is characterized by metabolic abnormalities including hyperuricemia, hyperphosphatemia, hyperkalemia, and hypocalcemia. These metabolic impairments may lead to renal insufficiency, cardiac arrhythmias, and potentially death (2). Guidelines for the management of TLS were published in 2008 (3), and an expert TLS panel consensus released recommendations in 2010 (4). Prevention and prompt treatment of TLS are crucial; therefore, early recognition of patient condition and evaluation of the risk of developing TLS is important (5-7). TLS usually results from the rapid destruction of cancer cells due to the initiation of chemotherapy. Patients with malignancies that have a high proliferative rate or a large tumor burden, or are highly sensitive to anticancer treatments, have a particularly high risk of developing TLS. TLS is divided into laboratory TLS and clinical TLS. Patients are required to have two or more abnormalities of uric acid, potassium, or phosphorus to be defined as having laboratory TLS (4). Clinical TLS requires the presence of laboratory TLS in addition to one or more of the following significant clinical complications: renal insufficiency, cardiac arrhythmia/sudden death, or seizures $(4,8)$. If patients do not exhibit TLS, they are evaluated for the risk of developing TLS. The risk assessment is based on the type of malignancy, tumor burden, the type of therapy, and renal function of the host. Accordingly, patients are stratified into low-, intermediate-, and high-risk groups. 
Multiple myeloma is a clonal neoplasm formed by malignant plasma cells. TLS in patients with multiple myeloma is considered very rare because of the relatively slow proliferation and response of malignant cells to chemotherapy, and is, therefore, classified as a low-risk disease $(4,9)$. There have been significant advancements in the treatment of multiple myeloma in the past 10 years (10-12), and new classes and combinations of drugs, including proteasome inhibitors and immunomodulatory drugs (IMiDs), have led to increased survival of patients (13-15). The incidence of TLS has not thoroughly been evaluated in patients receiving new agents such as bortezomib and IMiDs, although their improved antimyeloma effects may increase the risk of developing TLS.

In the present study, the incidence of TLS was retrospectively evaluated in patients with symptomatic multiple myeloma receiving chemotherapy at our Institution. The chemotherapeutic modalities included bortezomib, IMiDs, and conventional chemotherapies. The incidence of TLS was compared among different treatments. The risk factors for developing TLS were also sought.

\section{Patients and Methods}

Patients. This single-center retrospective study was conducted to assess the incidence of TLS in patients with multiple myeloma. This study was approved by the Ethics Committee of the University of Fukui (Fukui, Japan) (no. 2015064). All procedures performed in studies involving human participants were in accordance with the ethical standards of the Institutional Research Committee and with the 1964 Helsinki declaration and its later amendments or comparable ethical standards.

Patients who had been diagnosed with symptomatic multiple myeloma and underwent chemotherapy in the University of Fukui Hospital (Fukui, Japan) between April 2006 and December 2015 were evaluated. Symptomatic multiple myeloma was diagnosed according to the International Myeloma Working Group Criteria $(16,17)$. The cytogenetic risk was classified into standard risk and high risk, based on conventional cytogenetic studies or fluorescent in situ hybridization. Patients who had $17 \mathrm{p}-, \mathrm{t}(14 ; 16)$, or $\mathrm{t}(4 ; 14)$ were classified as the high-risk group. Normal cytogenetics and other cytogenetic abnormalities were classified as the standard risk group. Staging of multiple myeloma was by International staging system (ISS) based on the serum beta-2-microglobulin (BMG) and albumin levels at diagnosis (18). All the patients received chemotherapeutic regimens as the first cycle of induction treatment or re-induction treatment after disease relapse. The chemotherapy regimens included bortezomib plus dexamethasone (BD); melphalan plus prednisolone with bortezomib (MPB); thalidomide; lenalidomide plus dexamethasone (Rd); bortezomib plus lenalidomide with dexamethasone (VRD), melphalan plus prednisolone (MP), vincristine plus adriamicin with dexamethasone (VAD); and pomalidomide plus dexamethasone (POMDEX).

The diagnosis of TLS. TLS was diagnosed according to the criteria of Japanese TLS guidance (9). In brief, laboratory TLS is diagnosed if levels of two or more serum values of uric acid, potassium, or phosphorus are elevated beyond the corresponding upper limit of the normal range within 3 days before or 7 days after the initiation of treatment. Clinical TLS requires the presence of laboratory TLS in addition to one or more of the following significant clinical complications: renal insufficiency (serum creatinine $\geq 1.5 \times$ upper normal limit), cardiac arrhythmia/sudden death, or seizures.

Study end points. The primary objective was the incidence of TLS in the patients with symptomatic multiple myeloma undergoing chemotherapy. The risk factors that would predict the development of TLS were also sought among the tumor burden and the cytogenetics of multiple myeloma, the type of treatments, and serum parameters including renal function.

Statistical analyses. Graph generation and the statistical analyses were performed using GraphPad Prism software (version 6.0; GraphPad Software, Inc., San Diego, CA, USA). Values of $p<0.05$ were considered statistically significant.

\section{Results}

Patients' characteristics. The study population comprised of 64 patients (35 males, 29 females), with the median age of 71 years (Table I). The subtypes of monoclonal immunoglobulin (Ig) included $\operatorname{IgG}, \operatorname{Ig} \mathrm{A}$, and Bence-Jones. Cytogenetic analysis demonstrated that eight patients [two with deletion 17, eight with $\mathrm{t}(4 ; 14)]$ were classified into the high-risk group. One-third of the patients exhibited ISS III disease. A total of 124 chemotherapy courses were administered, among which 63 courses were bortezomib-based regimens and 34 courses were IMid-based regimens. All patients were classified into the lowrisk group for TLS according to the Japanese TLS guidance (9).

Development of TLS. All the patients received chemotherapeutic regimens as the first cycle of induction treatment or re-induction treatment after disease relapse. Among 124 chemotherapy courses, TLS occurred in 13 cases $(10.5 \%)$ with five cases of laboratory TLS (4.0\%) and eight of clinical TLS (6.5\%). Among them, five patients were positive for $\mathrm{t}(11 ; 14)$ by fluorescence in situ hybridization. From the viewpoint of the chemotherapeutic regimen, 11 cases of TLS (three laboratory and eight clinical TLS) occurred along with the treatment with bortezomibcontaining regimens (11/63 cases, 17.5\%) (Figure 1), while TLS occurred in two cases treated with non-bortezomibbased regimens (VAD, and MP) (2/61 cases, 3.2\%) ( $p=0.014$, Chi-squared test; Figure 1). Surprisingly, no TLS occurred in the patients treated with IMiD-containing regimens $(0 / 34$ cases, $0.0 \%)$.

The incidence of TLS was evaluated from the serum parameters at baseline (Figure 2). TLS developed more frequently in patients with elevated serum uric acid $(37.5 \%$ vs. $4.4 \%$ ), creatinine (25.5\% vs. $1.4 \%)$, or BMG (25.7\% vs. $4.5 \%)$, but not of potassium $(22.2 \% v s .9 .2 \%)$ and corrected calcium (10.3\% vs. 9.8\%) (Figure 2). Moreover, patients developing TLS demonstrated higher serum levels of uric 


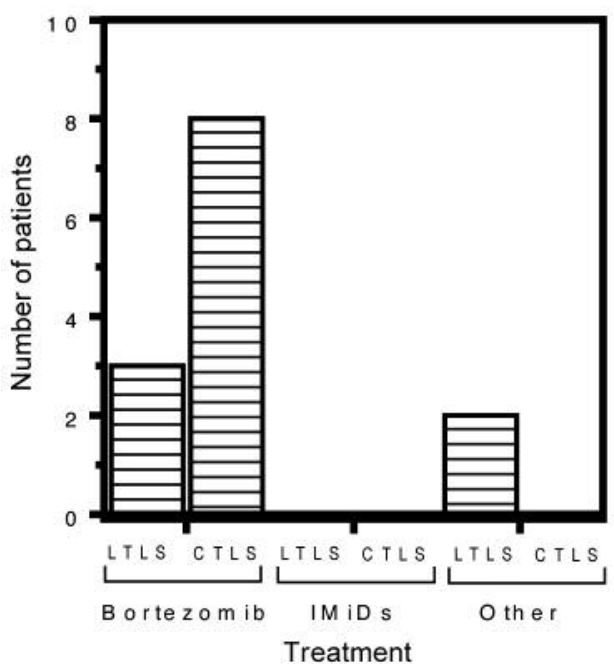

Figure 1. The incidence of tumor lysis syndrome among 120 chemotherapy courses in 64 patients with symptomatic myeloma, evaluated from the viewpoint of chemotherapy regimens. LTLS: Laboratory tumor lysis syndrome; CTLS: clinical tumor lysis syndrome; Bortezomib, bortezomib-containing regimens; IMiDs, regimens that included thalidomide, lenalidomide or pomalidomide; Other, other treatments including conventional melphalan with prednisolone, and vincristine with adriamicin and dexamethasone.

acid (median: $8.1 \mathrm{vs} .5 .4 \mathrm{mg} / \mathrm{dl}$ ), creatinine (median: $1.5 \mathrm{vs}$. $0.9 \mathrm{mg} / \mathrm{dl}$ ) and BMG (median: 11.2 vs. $3.9 \mathrm{mg} / \mathrm{dl}$ ), and lower eGFR (median: $33.5 \mathrm{ml} / \mathrm{min} v s .62 .0 \mathrm{ml} / \mathrm{min}$ ) than those who did not (Figure 3).

In the light of disease progression, the patients with advanced ISS [I/II: $3 / 64$ (4.7\%) vs. III: 10/33 (30.3\%)] or the presence of plasma cells in peripheral blood [positive: $4 / 15$, $26.7 \%$ vs. negative: $9 / 105(8.6 \%)$ ] also developed TLS more frequently (Figure 4).

Clinical TLS. Clinical TLS occurred in eight out of 124 chemotherapy courses $(6.5 \%)$. All these patients received bortezomib-containing regimens $(8 / 63,12.7 \%)$ (Table II; Figure 1). The patients exhibiting elevated baseline uric acid $(20.8 \%$ vs. $3.3 \%)$ or creatinine $(14.9 \%$ vs. $0 \%)$ developed clinical TLS more often than those who did not (Figure 5). Importantly, the incidence of clinical TLS was $33.3 \%$ when patients had renal dysfunction at baseline and were subsequently treated with bortezomib-based regimens (8/24 cases).

\section{Discussion}

The present study retrospectively analyzed the incidence of TLS in patients with symptomatic multiple myeloma at our Institution. TLS occurred in $10.5 \%$ of all chemotherapy courses, with $4.0 \%$ laboratory TLS and $6.5 \%$ clinical TLS
Table I. Patient characteristics.

\begin{tabular}{lc}
\hline & Number \\
\hline Number of patients & 64 \\
Median age (range), years & $(46-87)$ \\
Male/female & $35 / 29$ \\
Immunoglobulin & \\
IgG & 35 \\
IgA & 12 \\
Bence-Jones & 15 \\
Non-secretary & 2 \\
Cytogenetic karyotype & \\
Normal & \\
Deletion 17 & 2 \\
t (11;14) & 6 \\
t(14;16) & 0 \\
t(4;14) & 6 \\
ISS & \\
I & 12 \\
II & 23 \\
III & 24 \\
ND & 5 \\
Chemotherapy & \\
Total courses administered & 124 \\
Bortezomib-based & 63 \\
Lenalidomide-based & 23 \\
Thalidomide-based & 10 \\
Pomalidomide-based & 1 \\
Other (MP/VAD/Dex) & $27(15 / 8 / 4)$ \\
\hline
\end{tabular}

MP, Melphalan + prednisolone; VAD, vincristine + adriamycin + dexamethasone; DEX, dexamethasone; ND, not determined; BMG, beta-2-microgloburin; eGFR, estimated glomerular filtration rate.

(Table I). The incidence of TLS was $17.5 \%$ for bortezomibcontaining regimens, while no TLS occurred in the patients treated with IMiD-containing regimens (Figure 1). TLS developed more frequently in patients with hyperuricemia, renal dysfunction, or advanced disease (Figures 2-4). The incidence of clinical TLS was $33.3 \%$ when patients had renal dysfunction and were subsequently treated with bortezomibbased regimens (Figure 5).

TLS is thought to be rare in multiple myeloma because the antimyeloma activity of conventional chemotherapy (MP regimen) is insufficient to induce enough cytoreduction to develop it (13). Japanese TLS guidance classifies multiple myeloma as a low risk disease for the development of TLS (9). But the risk may increase if the case has a high myeloma cell percentage in bone marrow, or the presence of myeloma cells in peripheral blood, or del (13), and receives treatments using new agents (9). New agents such as proteasome inhibitors and IMiDs have been employed in first-line treatment regimens, that have improved significantly the prognosis of symptomatic myeloma (19). The frequency of TLS may, therefore, increase because of the greater 
A

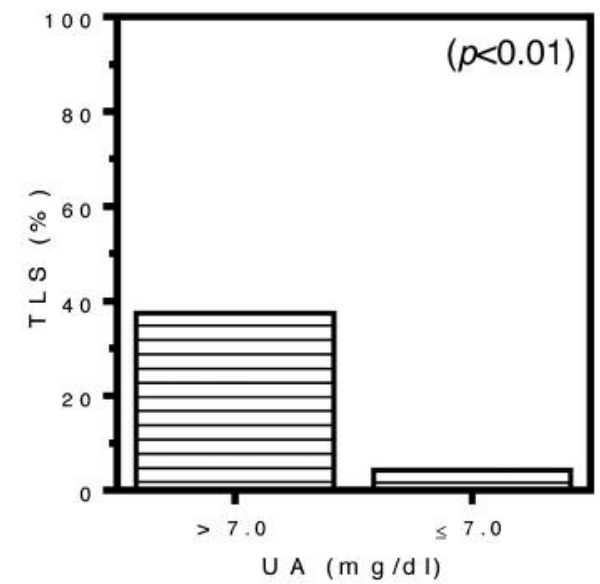

C

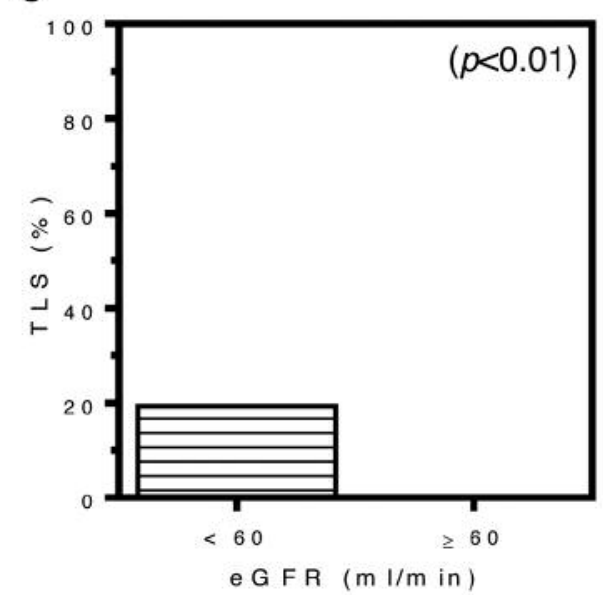

E

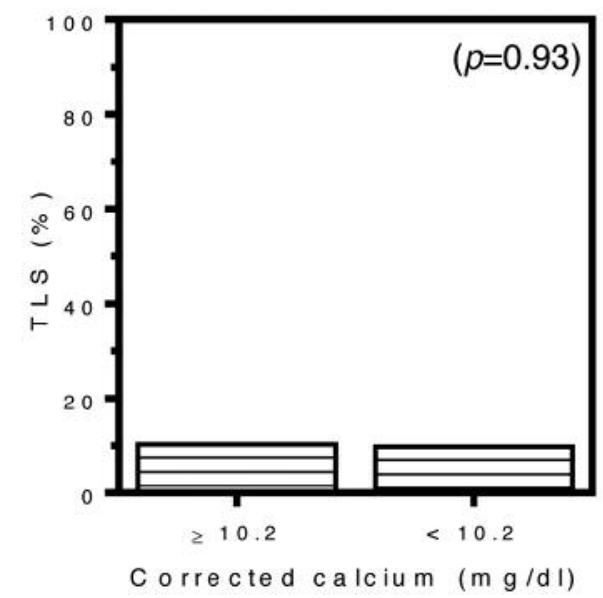

B

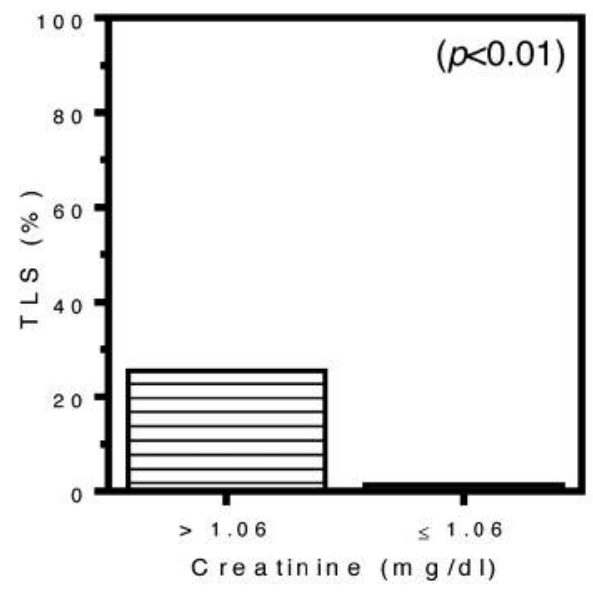

D

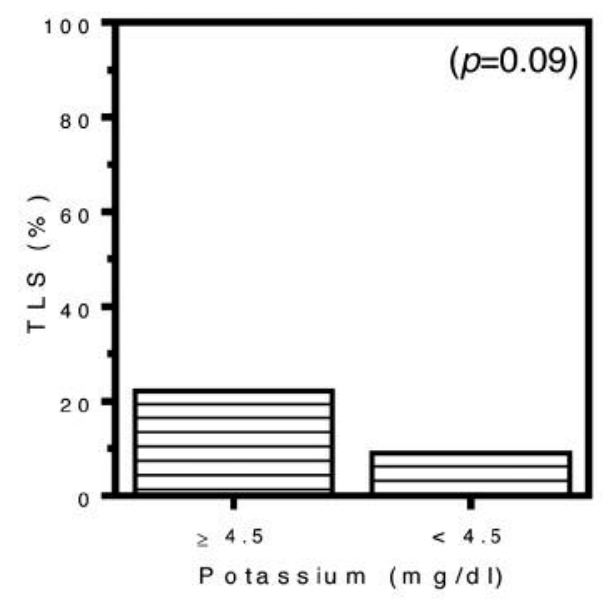

$\mathrm{F}$

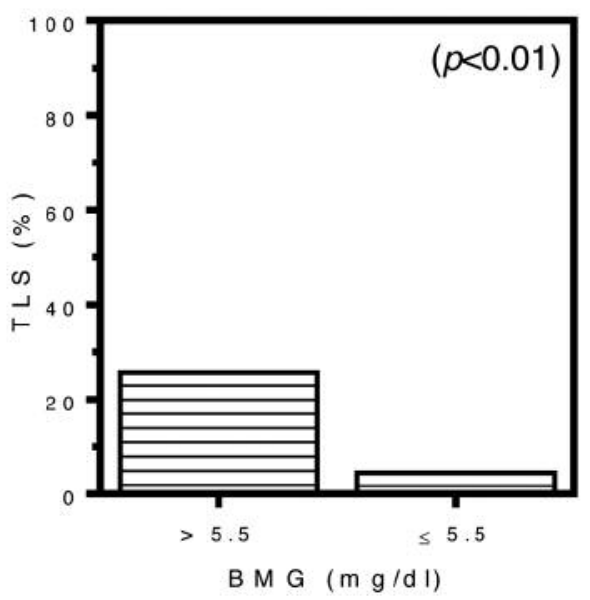

Figure 2. The incidence of tumor lysis syndrome (TLS) was evaluated from the serum values of uric acid (UA), creatinine, estimated glomerular filtration rate (eGFR), potassium, corrected calcium and beta-2-microglobulin (BMG) at baseline. A: $n=24$ cases with serum UA $>7.0 \mathrm{mg} / \mathrm{dl}$ (upper normal limit), $n=91$ cases with serum $U A \leq 7.0 \mathrm{mg} / \mathrm{dl}$. B: $n=47$ cases with serum creatinine $>1.06 \mathrm{mg} / \mathrm{dl}, \mathrm{n=73}$ cases with the serum creatinine $\leq 1.06 \mathrm{mg} / \mathrm{dl}$. C: $n=67$ cases with eGFR $<60 \mathrm{ml} / \mathrm{min}$ (lower normal limit), $n=53$ cases with the eGFR $\geq 60 \mathrm{ml} / \mathrm{min}$. $D: n=18$ cases with the serum potassium $\geq 4.5 \mathrm{mEq} / \mathrm{l}$ (upper normal limit), $n=102$ cases with the serum potassium $<4.5 \mathrm{mEq} / \mathrm{l}$. E: $n=58$ cases with corrected calcium $\geq 10.2 \mathrm{mg} / \mathrm{dl}$ (upper normal limit), $n=61$ cases with corrected calcium $<10.2 \mathrm{mg} / \mathrm{dl}$. $F: n=35$ cases with serum BMG $>5.5 \mathrm{mg} / \mathrm{dl}$ (upper normal limit), $n=66$ cases with serum $B M G \leq 5.5 \mathrm{mg} / \mathrm{dl}$. Chi-squared test was used for statistical analyses. 
A

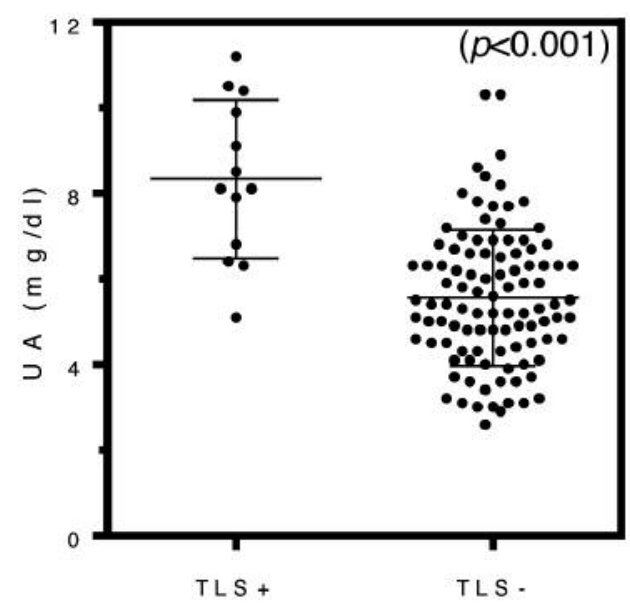

C

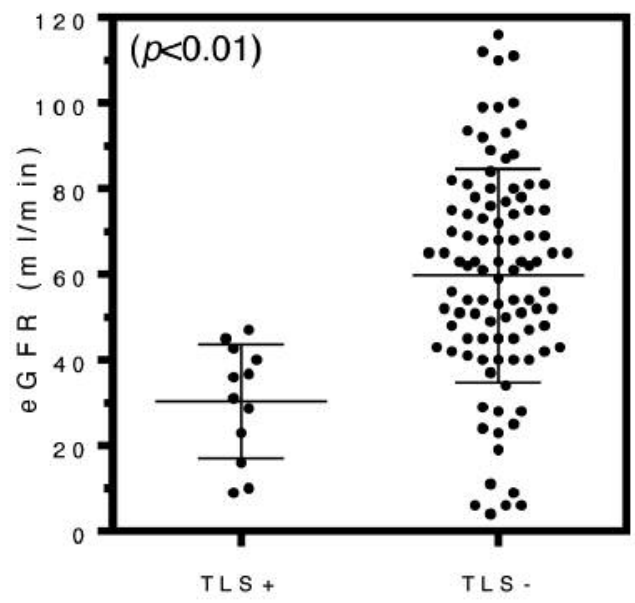

E

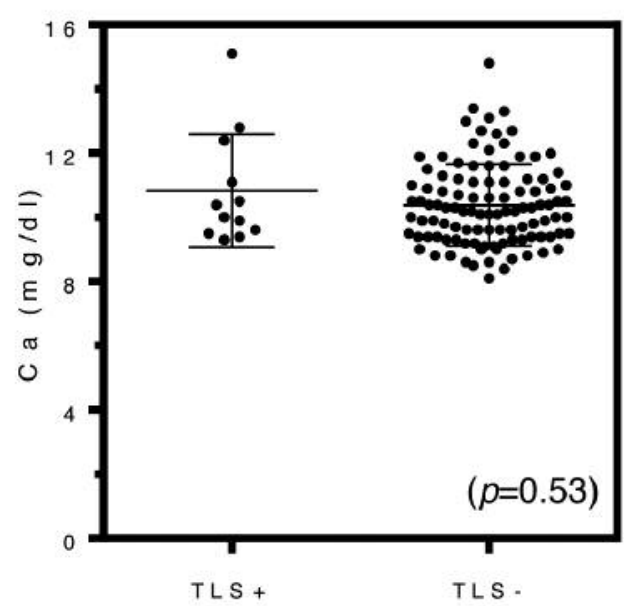

B

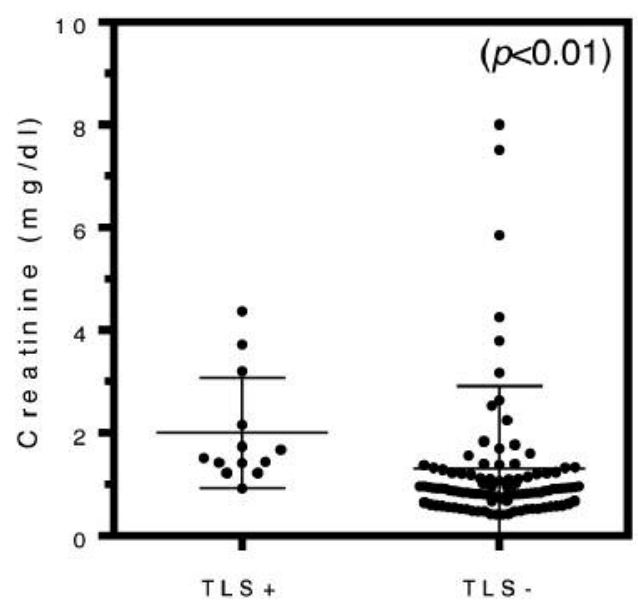

D

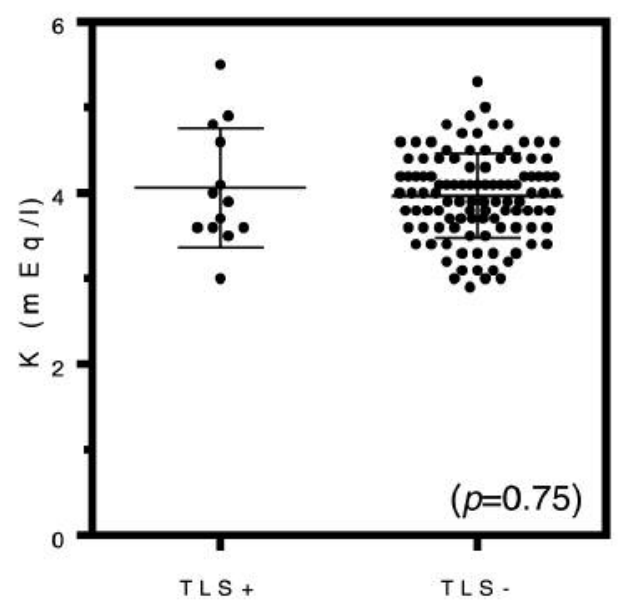

$\mathrm{F}$

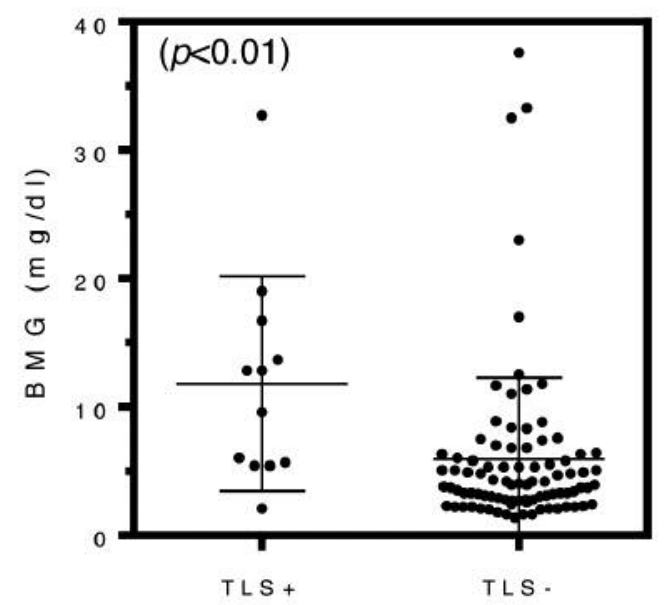

Figure 3. The comparison of the laboratory findings at baseline between the patients who developed tumor lysis syndrome (TLS+) and those who did not (TLS-). A: Serum uric acid. B: Serum creatinine. C: Estimated glomerular filtration rate. D: Serum potassium. E: Serum corrected calcium. F: Serum beta-2-microgloburin (BMG). Mann-Whitney U-test was used for statistical analyses. 
A

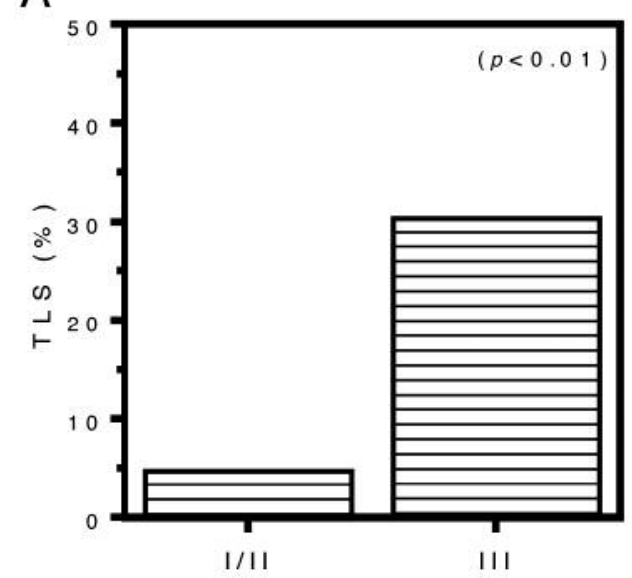

B

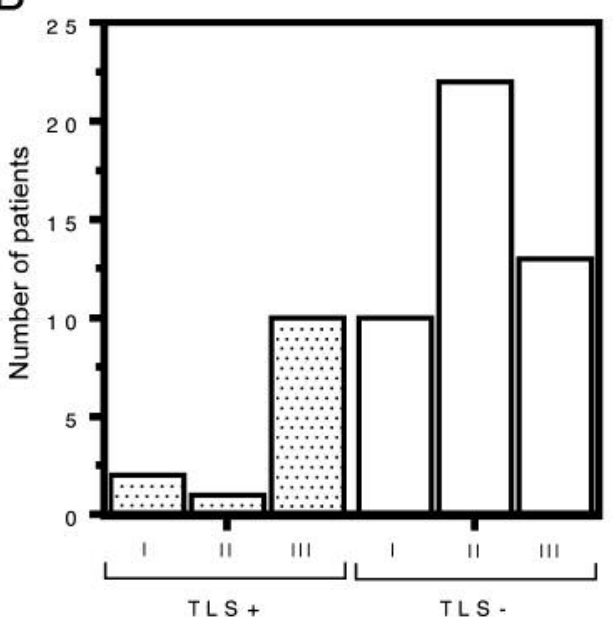

C

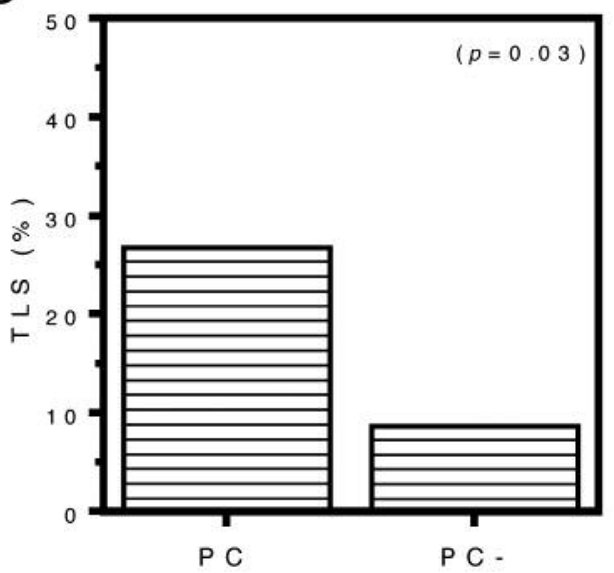

Figure 4. A: The relationship between the incidence of tumor lysis syndrome (TLS) and International Staging System (ISS) class. B: The ISS distribution in the patients with TLS (TLS+) and those without (TLS-). $n=64$ with ISS I/II, n=33 with ISS III. Chi-squared test was used for statistical analyses. C: The incidence of TLS according to the presence of plasma cells in peripheral blood. $n=15$ patients with plasma cells $(P C+)$ in peripheral blood, $n=105$ patients without PC. Chisquared test was used for statistical analyses.
A

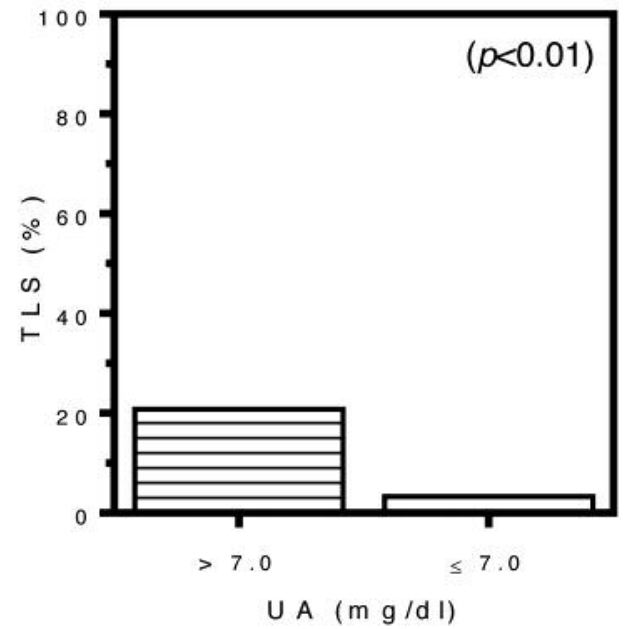

B

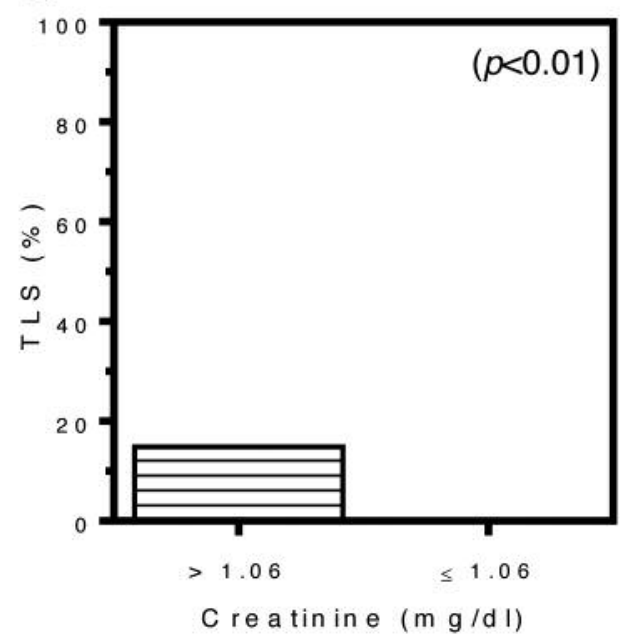

Figure 5. The incidence of clinical tumor lysis syndrome (TLS) was evaluated according to the serum values of uric acid (UA) (A) and creatinine $(B)$. $A: n=24$ cases with serum $U A>7.0 \mathrm{mg} / \mathrm{dl}$ (upper normal limit), $n=91$ cases with serum $U A \leq 7.0 \mathrm{mg} / \mathrm{dl}$. $B: \mathrm{n}=47$ cases with serum creatinine $>1.06 \mathrm{mg} / \mathrm{dl}, n=73$ cases with serum creatinine $\leq 1.06 \mathrm{mg} / \mathrm{dl}$. Chi-squared test was used for statistical analyses.

cytotoxicity of new agents and an awareness of TLS after the introduction of TLS guidelines.

The incidence of TLS associated with multiple myeloma has seldom been evaluated in large-scale studies after TLS guidelines were published. Sezer et al. reported eight episodes of TLS in seven patients with bortezomib-based treatment among 496 patients (20). Biochemical abnormalities resolved with supportive therapy in six patients but proved fatal in one patient (20). Suzuki et al. investigated parameters that could predict the development of TLS in patients undergoing bortezomib treatment (21). They retrospectively reviewed 35 
Table II. Characteristics of patients developing clinical tumor lysis syndrome.

\begin{tabular}{|c|c|c|c|c|c|}
\hline Age, gender/regimen & Creatinine $(\mathrm{mg} / \mathrm{dl})$ & $\mathrm{K}(\mathrm{mEq} / \mathrm{l})$ & Uric acid (mg/dl) & BMG (mg/dl) & $\mathrm{P}(\mathrm{mg} / \mathrm{dl})$ \\
\hline $77 \mathrm{M} / \mathrm{Bd}$ & 1.68 & 5.5 & 6.8 & 5.7 & 4.5 \\
\hline $74 \mathrm{M} / \mathrm{MPB}$ & 1.22 & 4.1 & 10.5 & 12.8 & 5.8 \\
\hline $69 \mathrm{M} / \mathrm{Bd}$ & 1.41 & 3.6 & 11.2 & 13.7 & 4.3 \\
\hline $71 \mathrm{~F} / \mathrm{Bd}$ & 1.74 & 3.7 & 9.9 & ND & 3.7 \\
\hline $65 \mathrm{~F} / \mathrm{Bd}$ & 4.37 & 4.0 & 7.9 & 16.7 & 4.9 \\
\hline $65 \mathrm{M} / \mathrm{BCD}$ & 3.20 & 3.9 & 10.4 & 19.0 & 4.1 \\
\hline $84 \mathrm{~F} / \mathrm{Bd}$ & 3.72 & 3.5 & 6.4 & 32.7 & 4.3 \\
\hline $56 \mathrm{M} / \mathrm{Bd}$ & 1.42 & 3.6 & 6.3 & 5.4 & 3.1 \\
\hline
\end{tabular}

M: Male; F: female; BMG, beta-2-microgloburin; Bd, bortezomib+dexamethasone; MPB, melphalan+prednisolone+bortezomib; BCD, bortezomib+cycrophosphamide+dexamethasone; ND: not determined.

patients with relapsed or refractory myeloma treated with bortezomib-containing regimens. They revealed that clinical TLS occurred in six patients $(17.1 \%)$ during the first course of bortezomib-containing treatment among all the patients. They found that TLS occurred more often in patients with rapidly progressive anemia. The incidence of clinical TLS in our study was compatible with theirs $(12.7 \%)$ (Table II, Figure 1). There have been several case reports regarding TLS associated with multiple myeloma. Terpos et al. reported a 34-year-old man with stage IIIA IgG-K myeloma who relapsed after autologous stem cell transplantation (22). At baseline, nearly $100 \%$ of the bone marrow cells were replaced by plasma cells, and his serum creatinine and uric acid levels were $85 \mu \mathrm{mol} / 1$ and $0.44 \mathrm{mmol} / \mathrm{l}$, respectively. He was treated with bortezomib at a dose of $1.3 \mathrm{mg} / \mathrm{m}^{2}$ twice weekly for 2 weeks in a 3 -week cycle. TLS arose after the second dose of bortezomib. The next dose of the agent was delayed and symptomatic treatment was successfully performed. Dhanraj et al. reported a 65-year-old man with ISS III multiple myeloma (23). Thirty percent of bone marrow cells were occupied with plasma cells. His serum creatinine level was $1.3 \mathrm{mg} / \mathrm{dl}$. He developed clinical TLS $48 \mathrm{~h}$ after the administration of bortezomib $\left(1.3 \mathrm{mg} / \mathrm{m}^{2}\right)$ and dexamethasone (40 mg/body) in combination. The patient of this latter study died despite intensive treatments including hemodialysis and mechanical ventilation. TLS was also reported to be found in patients who were treated with thalidomide (24-26). Thus, including our present investigation, TLS appears to have occurred more frequently in recent years than before in patients with myeloma, since patients have been treated more aggressively with new agents.

The present study demonstrated that TLS develops more frequently in patients with elevated serum uric acid, creatinine, BMG, and with advanced ISS or the presence of plasma cells in peripheral blood (Figures 2 and 4). These determinants were compatible with those previously pointedout as risk factors. Moreover, one thing to be emphasized is that all the patients presenting clinical TLS were treated with bortezomib, while no patient treated with IMiDs developed laboratory and clinical TLS (Figure 1). TLS risk may be higher for treatment with bortezomib.

The development of TLS associated with multiple myeloma has not yet been investigated thoroughly after the introduction of new agents and the publication of TLS guidelines. The present study conclusively demonstrates the high risk of TLS in patients with renal dysfunction receiving bortezomib.

\section{Conflicts of Interest}

All Authors declare they have no conflict of interest in regard to this study.

\section{Acknowledgements}

This work was supported in part by a grant from the Gout Research Foundation, Tokyo, Japan (2013, No. H26-3-5).

\section{References}

1 Firwana BM, Hasan R, Hasan N, Alahdab F, Alnahhas I, Hasan $\mathrm{S}$ and Varon J: Tumor lysis syndrome: a systematic review of case series and case reports. Postgrad Med 124: 92-101, 2012.

2 Cohen LF, Balow JE, Magrath IT, Poplack DG and Ziegler JL: Acute tumor lysis syndrome. A review of 37 patients with Burkitt's lymphoma. Am J Med 68: 486-491, 1980.

3 Coiffier B, Altman A, Pui CH, Younes A and Cairo MS: Guidelines for the management of pediatric and adult tumor lysis syndrome: an evidence-based review. J Clin Oncol 26: 2767-2778, 2008.

4 Cairo MS, Coiffier B, Reiter A and Younes A: TLS Expert Panel. Recommendations for the evaluation of risk and prophylaxis of tumour lysis syndrome (TLS) in adults and children with malignant diseases: an expert TLS panel consensus. Br J Haematol 149: 578-586, 2010.

5 Takai M, Yamauchi T, Fujita K, Lee S, Ookura M, Kishi S, Urasaki Y, Yoshida A, Iwasaki H and Ueda T: Controlling serum uric acid using febuxostat in cancer patients at risk of tumor lysis syndrome. Oncol Lett 8: 1523-1527, 2014. 
6 Takai M, Yamauchi T, Ookura M, Matsuda Y, Tai K, Kishi S, Yoshida A, Iwasaki H, Nakamura T and Ueda T: Febuxostat for management of tumor lysis syndrome including its effects on levels of purine metabolites in patients with hematological malignancies. - A single institution's, pharmacokinetic and pilot prospective study. Anticancer Res 34: 7287-7296, 2014.

7 Takai M, Yamauchi T, Matsuda Y, Tai K, Ikegaya S, Kishi S, Urasaki Y, Yoshida A, Iwasaki $\mathrm{H}$ and Ueda T: Reduced administration of rasburicase for tumor lysis syndrome: A singleinstitution experience. Oncol Lett 9: 2119-2125,2015.

8 Cairo MS and Bishop M: Tumour lysis syndrome: new therapeutic strategies and classification. Br J Haematol 127: 3 $11,2004$.

9 Ishizawa K, Kirito K, Kawano T, Tokuhira M, Nagai H, Yuza Y and You K: Tumor Lysis Syndrome Clinical Guidance. Kanehara, Tokyo, 2013.

10 Rajkumar SV and Kumar S: Multiple myeloma: diagnosis and treatment. Mayo Clin Proc 91: 101-119, 2016.

11 Sonneveld P and Broijl A: Treatment of relapsed and refractory multiple myeloma. Haematologica 101: 396-406, 2016.

12 Lehners N, Hayden PJ, Goldschmidt H and Raab MS: Management of high-risk myeloma: an evidence-based review of treatment strategies. Expert Rev Hematol 9: 753-765, 2016.

13 Naymagon L and Abdul-Hay M: Novel agents in the treatment of multiple myeloma: a review about the future. J Hematol Oncol 9: 52, 2016.

14 Redic KA, Hough SM and Price EM: Clinical developments in the treatment of relapsed or relapsed and refractory multiple myeloma: impact of panobinostat, the first-in-class histone deacetylase inhibitor. Onco Targets Ther 9: 2783-2793, 2016.

15 Libby E, Garcia D, Quintana D, Fekrazad MH, Bauman J, Ebaid A, Hromas R, Rabinowitz I and Wiggins C: Disease-specific survival for patients with multiple myeloma: significant improvements over time in all age groups. Leuk Lymphoma 55: 2850-2857, 2016

16 Durie BG, Kyle RA, Belch A, Bensinger W, Blade J, Boccadoro M, Child JA, Comenzo R, Djulbegovic B, Fantl D, Gahrton G, Harousseau JL, Hungria V, Joshua D, Ludwig H, Mehta J, Morales AR, Morgan G, Nouel A, Oken M, Powles R, Roodman D, San Miguel J, Shimizu K, Singhal S, Sirohi B, Sonneveld P, Tricot G and Van Ness B: Scientific Advisors of the International Myeloma Foundation. Myeloma management guidelines: a consensus report from the Scientific Advisors of the International Myeloma Foundation. Hematol J 4: 379-398, 2003.

17 Rajkumar SV, Dimopoulos MA, Palumbo A, Blade J, Merlini G, Mateos MV, Kumar S, Hillengass J, Kastritis E, Richardson P, Landgren O, Paiva B, Dispenzieri A, Weiss B, LeLeu X, Zweegman S, Lonial S, Rosinol L, Zamagni E, Jagannath S, Sezer O, Kristinsson SY, Caers J, Usmani SZ, Lahuerta JJ, Johnsen HE, Beksac M, Cavo M, Goldschmidt H, Terpos E, Kyle RA, Anderson KC, Durie BG and Miguel JF: International Myeloma Working Group updated criteria for the diagnosis of multiple myeloma. Lancet Oncol 15: e538-548, 2014.
18 Greipp PR, San Miguel J, Durie BG, Crowley JJ, Barlogie B, Bladé J, Boccadoro M, Child JA, Avet-Loiseau H, Kyle RA, Lahuerta JJ, Ludwig H, Morgan G, Powles R, Shimizu K, Shustik C, Sonneveld P, Tosi P, Turesson I and Westin J: International staging system for multiple myeloma. J Clin Oncol 23: 3412-3420, 2005.

19 Moreau P, Attal M and Facon T: Frontline therapy of multiple myeloma. Blood 125: 3076-3084, 2015.

20 Sezer O, Vesole DH, Singhal S, Richardson P, Stadtmauer E, Jakob C, Boral AL, Esseltine DL and Mehta J: Bortezomibinduced tumor lysis syndrome in multiple myeloma. Clin Lymphoma Myeloma 7: 233-235, 2006.

21 Suzuki K, Terui Y, Nishimura N, Ueda K, Mishima Y, Sakajiri $\mathrm{S}$, Yokoyama M, Aiba K and Hatake K: Rapid progression of anemia related to tumor lysis syndrome associated with bortezomib treatment in myeloma patients. Jpn J Clin Oncol 44: 435-441, 2014

22 Terpos E, Politou M and Rahemtulla A: Tumour lysis syndrome in multiple myeloma after bortezomib (VELCADE) administration. J Cancer Res Clin Oncol 130: 623-625, 2004.

23 Dhanraj KM and Biswajit D: Tumor lysis syndrome in multiple myeloma treated with bortezomib. J Pharmacol Pharmacother 5: 161-162, 2014.

24 Cany L, Fitoussi O, Boiron JM and Marit G: Tumor lysis syndrome at the beginning of thalidomide therapy for multiple myeloma. J Clin Oncol 208: 2212, 2002.

25 Fuente N, Mañe JM, Barcelo R, Muñoz A, Perez-Hoyos T and Lopez-Vivanco G: Tumor lysis syndrome in a multiple myeloma treated with thalidomide. Ann Oncol 15: 537, 2004.

26 Huston A, Brown J and Roodman GD: Tumor lysis syndrome following thalidomide and dexamethasone therapy for newly diagnosed multiple myeloma. Exp Hematol 34: 1616, 2016.
Received September 28, 2016

Revised October 19, 2016

Accepted October 20, 2016 